\title{
Social Cognition Impairments in Mice Overexpressing Alpha-Synuclein Under the Thy1 Promoter, a Model of Pre-manifest Parkinson's Disease
}

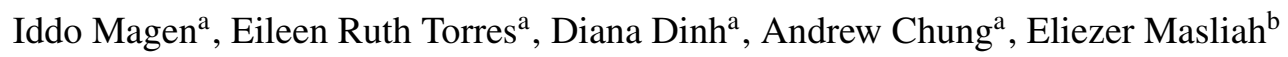 \\ and Marie-Françoise Chesselet ${ }^{\mathrm{a}, *}$ \\ ${ }^{a}$ Department of Neurology, The David Geffen School of Medicine at UCLA, Los Angeles, CA, USA \\ ${ }^{\mathrm{b}}$ Department of Neuroscience, University of California, San Diego School of Medicine, La Jolla, CA, USA
}

\begin{abstract}
.
Background: Patients with Parkinson's disease (PD) may exhibit deficits in "Theory of Mind", the ability to read others' mental states and react appropriately, a prerequisite for successful social interaction. Alpha-synuclein overexpression is widely distributed in the brain of patients with sporadic PD, suggesting that it may contribute to the non-motor deficits observed in PD patients. Mice over-expressing human wild-type alpha-synuclein under the Thy1 promoter (Thy1-aSyn mice) have synaptic deficits in the frontostriatal pathway, low cortical acetylcholine, and high level of expression of mGluR5 receptors, which have all been implicated in social recognition deficits.

Objective: To determine whether Thy1-aSyn mice present alterations in their response to social stimuli.

Methods: We have submitted Thy1-aSyn mice to tests adapted from autism models.

Results: At 7-8 month of age Thy1-aSyn mice explored their conspecifics significantly less than did wild-type littermates, without differences in exploration of inanimate objects, and pairs of Thy1-aSyn mice were involved in reciprocal interactions for a shorter duration than wild-type mice at this age. These deficits persisted when the test animal was enclosed in a beaker and were not present at 3-4 months of age despite the presence of olfactory deficits at that age, indicating that they were not solely caused by impairment in olfaction.

Conclusion: Thy1-aSyn mice present progressive deficits in social recognition, supporting an association between alphasynuclein overexpression and Theory of Mind deficits in PD and providing a useful model for identifying mechanisms and testing novel treatments for these deficits which impact patients and caretakers quality of life.
\end{abstract}

Keywords: Theory of mind, mGluR5, autistic disorder

\section{NON-STANDARD ABBREVIATIONS}

ASD

mGluR5

PD
Autistic Spectrum Disorders

metabotropic glutamate receptor 5

Parkinson's disease

\footnotetext{
*Correspondence to: Marie-Françoise Chesselet, M.D., Ph.D., Department of Neurology, The David Geffen School of Medicine at UCLA, 710 Westwood Plaza, Los Angeles, CA 90095-1769, USA. Tel.: +1 310267 1781; Fax: +1 310267 1786; E-mail: mchesselet@mednet.ucla.edu.
}

$\begin{array}{ll}\text { Thy 1-aSyn } & \text { mice overexpressing alpha-synuclein } \\ & \text { under the Thy } 1 \text { promoter } \\ \text { ToM } & \text { theory of mind } \\ \text { WT } & \text { wild type }\end{array}$

\section{INTRODUCTION}

Patients with Parkinson's disease (PD) may exhibit a variety of non-motor symptoms, including olfactory, 
autonomic and cognitive deficits as well as sleep and affective disorders such as depression and anxiety, which may even precede the characteristic motor deficits $[1,2]$. Related to the cognitive deficits, though different in nature, impairments in advanced Theory of Mind (ToM), refer to the ability to infer other people's thoughts, intentions, or emotions. ToM is a basic skill necessary for maintaining appropriate social interactions which is markedly impaired primarily in autism and autistic spectrum disorders (ASD) [3]. Although evidently much milder than in autistic patients, ToM deficits were also described in PD patients at early stages of disease [4-6]. Specifically, two subcomponents of ToM were impaired in early PD: an affective subcomponent, which refers to beliefs about other people's feelings, and a cognitive subcomponent, which refers to beliefs about other people's beliefs. ToM impairments impact the social interactions of the patients and, as a result, likely affect their quality of life and that of their caretakers.

ToM impairments are found in non-demented PD patients [5], and are not improved by dopaminergic treatments [4], suggesting that their mechanisms differ from those leading to severe cognitive deficits and motor impairments. Rather, since ToM is mediated by frontostriatal "loops" [7], frontostriatal abnormalities may be causing ToM impairments in PD [8-12].

Extensive studies have attempted to evaluate the equivalent of a ToM in mice because of the relevance of deficits in ToM to autism spectrum disorders. The most successful approach has been to measure "social cognition", an aspect of ToM. This can be achieved in mice by evaluating the interest of the animal for congeners versus inanimate objects, and several tests were developed that showed deficits in mouse models of autism [13]. We have taken this approach to evaluate ToM in a genetic mouse model of early stage PD.

Alpha-synuclein is related genetically to familial forms of PD and to an increased risk and severity of sporadic PD [14, 15]. Furthermore, misfolded alphasynuclein is a major component of Lewy bodies, the characteristic pathological lesions present in the brains of patients with PD $[16,17]$. Alpha-synuclein pathology is widespread in both the central and peripheral nervous system in PD and may be responsible for the wide variety of non-motor deficits occurring in the disease [18, 19].

In a mouse model expressing human wild-type alpha-synuclein under the Thy1 promoter (Thy1-aSyn mice), alpha-synuclein over-expression is sufficient to produce deficits in olfaction, sleep, autonomic, and digestive function [20-22] and, perhaps most relevant to theory of mind deficits, cognitive dysfunction in a wide range of tasks [23]. Mechanistically, these mice show alterations in the corticostriatal pathway [24, 25] and overexpression of mGluR5 receptors [26]. Corticostriatal dysfunction is associated with ASD [27] and negative allosteric modulation of the mGluR5 receptors rescued social behavior in ASD models [28]. Together, these observations led us to test the hypothesis that alpha-synuclein overexpressing mice also exhibit deficits in social behavior.

We show that profound social recognition deficits were present in the Thy1-aSyn mice at 7-8 but not 3-4 months of age. The deficits were independent from impairment in activity or olfaction. The data indicate that alpha-synuclein over-expression can lead to deficits reminiscent of ToM anomalies observed in PD patients.

\section{MATERIALS AND METHODS}

Mice

Animal care was in accordance with the United States Public Health Service Guide for the Care and Use of Laboratory Animals, and procedures were approved by the Institutional Animal Care and Use Committee at the University of California Los Angeles (UCLA). Except for the target mice in the social approach task, which were males from the $129 / \mathrm{SvJ}$ strain (see description of this test below), all mice used for this study were transgenic mice overexpressing human wild type alpha-synuclein under the Thy-1 promoter (Thy1-aSyn) and their wild-type (WT) littermates. Thy1-aSyn mice were developed and crossed into a hybrid C57BL/6-DBA/2 background as described before [29], by mating N7 female hemizygous for the transgene with male WT mice on the hybrid background obtained from Charles River Laboratories, Inc. [Wilmington, MA;[30-32]]. Male and female mice from the same litters were never bred together. Only male mice were used to avoid inconsistencies due to random inactivation of the $\mathrm{x}$ chromosomes (in which the transgene is inserted) in females. Male mice from 19 litters were included in the study. Mice were genotyped at the beginning of the study and their genotypes were confirmed by polymerase chain reaction (PCR) amplification analysis of tail DNA at the end of the experiment. Between one and four animals were housed in each cage. Cages were 7.5 inches (height) $\times 11.5$ inches (length) $\times 5$ inches (width) in size, and contained Sani chip bedding (Newco, Montville, NJ) and nesting squares 
Table 1

Details of all the cohorts of mice used in this study

\begin{tabular}{lcccc}
\hline Cohort $^{\#}$ & Number of mice & Number of litters & $\begin{array}{c}\text { Time points tested at } \\
\text { social reciprocal interaction task }\end{array}$ & $\begin{array}{c}\text { Time points tested } \\
\text { at social approach task }\end{array}$ \\
\hline 1 & 11 WT, 13 Thy1-aSyn & 5 & $3-4$ and 7-8 months & $3-4$ months \\
2 & 12 WT, 10 Thy1-aSyn & 6 & $7-8$ months & $7-8$ months \\
3 & 10 WT, 10 Thy1-aSyn & 8 & $3-4$ months & Not tested \\
\hline
\end{tabular}

( 2 inches $\times 2$ inches) made of cotton for enrichment. Mice were fed on NIH-31 modified mouse/rat sterilizable diet (Harlan) and maintained on a reverse light/dark cycle with lights off at $10 \mathrm{am}$ and all testing was performed between 12 and 4 pm during the dark cycle under dim light. Food and water were available ad libitum.

Three cohorts were tested in this study. The details of the different cohorts are summarized in Table 1.

\section{Social approach task}

Mice used as novel mice (also called target mice) to be explored by the subject mice were male mice from the 129/SvJ strain (Charles River Laboratories, Wilmington, MA), known for their docile nature. Target mice for each set were roughly the same age as the subject mice.

The social approach task was adapted from a previously reported procedure [13]. A three-chamber Plexiglas box divided into three adjacent chambers, each $20 \mathrm{~cm}$ (length) x $40.5 \mathrm{~cm}$ (width) x $22 \mathrm{~cm}$ (height), separated by two removable doors, was used for the experiments (made by Plastic Zone, Tarzana, CA). Steel wire pencil cups (from www.kitchen-plus.com, item $315,10.16 \mathrm{~cm}$ in diameter, $10.8 \mathrm{~cm}$ in height) were used as both containment for the target mice and as inanimate objects to be explored. In all phases requiring these cups, glass jars were used as weights placed on top of the wire cups to prevent mice from overturning them. The experiments were conducted in a dimly lit area during the dark phase of the mouse sleeping cycle to ensure high levels of activity. The brightness of the right and left chambers was measured with a light meter (Fisher Scientific, Pittsburgh, PA) and kept at $\sim 6.0 \pm 0.5$ lux before experiments were initiated, to ensure that mice have no baseline preference for either chamber due to differences in light intensity. Three sides of the box-the two short sides and the long side facing away from the experimenter-were covered to prevent mice from using spatial cues, and the long side facing the experimenter was left open for experimenter view.

\section{Experiment 1: Using wire cups as containments}

After habituation of one hour to the testing room in the home cage, target mice from the $129 / \mathrm{SvJ}$ strain were placed inside the wire cup in one of the side chambers for three 10-min sessions on the day prior to the test, to habituate them to the apparatus and the pencil cup. Two mice were habituated at a time, with one in each side chamber. This part of the experiment was not recorded, but mice were watched for disruptive behaviors such as bar-biting, circling or excessive selfgrooming. None of the mice displayed these behaviors during the habituation to the cup.

The next day, each subject mouse was tested in an experiment with three phases, each 10-min long (measured with a simple timer): I and II, the habituation phases, and III, the experimental phase. In phase I, the subject mouse was habituated to the center chamber, with no access to the side chambers. In phase II, the separating doors were removed to allow the mouse free exploration of all three chambers. Two silent stopwatches were used to measure the time the mouse spent in the right and left chambers, to ensure lack of side preference. This phase was recorded with a video camera.

The mouse was then guided back to the center chamber and the doors replaced. In phase III, an empty wire cup was placed in the center of the right or left chamber as the novel object and the cup containing the target mouse was placed in the center of the other chamber. Location of the novel object and the novel mice were counterbalanced to avoid confounding effect of side preference. Weights (glass jars) were placed on top of each cup to prevent overturning. The doors were then removed and the timer for 10-min started. During this phase, the experimenter timed how long the subject mouse explored the empty wire cup and the wire cup with the novel mouse, using two silent stopwatches. After testing each subject mouse, the three-chamber apparatus was thoroughly cleaned with ethanol, and the wire cups were replaced with new ones.

This phase was also recorded, and exploration times rated on-line were confirmed by rating the videos offline by a different rater unaware of genotype. The time 
spent in the chamber and the numbers of entries to each chamber were also rated off-line.

\section{Experiment 2: Using glass beakers as containments}

An additional experiment was performed using glass, airtight 1liter PYREX beakers (Corning Inc., Corning, NY) instead of wire cups to prevent olfactory detection, with the same setup as phase III described above. Since the mice were already habituated to the apparatus, phases I and II were not repeated. This experiment was done at least three days after the end of the experiment with the wire cups to ensure that mice did not lose interest in the test as a result of frequent and repeated testing. After testing each subject mouse, the three-chamber apparatus was thoroughly cleaned with ethanol, and the beakers were replaced with new ones. This experiment was also recorded by video. The times the mouse spent in each chamber and the number of entries into each chamber in this experiment were also measured as in phase III to wholly account for any possible differences between Thy1-aSyn and wild-type mice.

Procedures for Experiment 2 with beakers were done as controls only for 7-8 month mice, as younger mice did not show any deficits using the wire cup as containment and it was not necessary to control for olfactory deficits in that case.

\section{Reciprocal social interaction}

In the reciprocal interaction task, two male WT or transgenic mice from different litters and different home cages, aged 3-4 months and 7-8 months were placed in a clean, standard cage and their activity was recorded on a video camera for 20 minutes per trial. Prior to the test, one of the mice was habituated to the clean cage for 20 minutes and at the end of this period the other partner was added. This protocol was shown to facilitate social interactions compared to when the two mice were put together [33].

The behaviors that were documented were: nose-tonose sniffing (both mice sniffing the nose/snout region of each other); front approach (moving toward the partner from a distance, in a head-on manner); push-crawl (pushing the head underneath the partner's body and/or squeezing between the wall/floor or crawling over or under the partner's body); wrestling; following (walking straight behind the partner, keeping pace with the one ahead). The number of bouts mice spent in each one of these behaviors was recorded. Total time spent in interaction was also measured off line, as well as the total time the mice spent sniffing each other. These are all behaviors that are common in wild-type mice, and they have been studied and found to be mildly impaired in the Shank 3 mutant mice, which is a mouse model of ASD [34].

\section{Statistical analysis}

In phase II of experiment 1 of the social approach task, the time spent in the left and right chambers was analyzed with two way repeated measures analysis of variance (ANOVA) with chamber as within-subject factor and genotype as between-subject factor, for each age separately. In phase III of experiment 1 , sniffing time of the empty wire cup and the wire cup containing the novel mouse, as well as time spent in, and the number of entries to the respective chambers were analyzed by two-way repeated measures ANOVA with variable (empty wire cup vs. wire cup with novel mouse, or the chambers containing them) as withinsubject factor and genotype as between subject factor, for each age separately. In experiment 2, these parameters were analyzed the same way, except that variables were empty beaker, beaker with novel mouse or the chambers containing them. Bonferroni test was used for post hoc comparisons. The data in the reciprocal interaction test were mostly analyzed using two ways ANOVA with age and genotype as independent factors followed by Bonferroni post hoc, but wrestling behavior was compared between the genotypes at each age by analyzing the frequency of mice displaying this kind of behavior, using Fisher's exact test. Power analysis was conducted to determine the minimal number of mice required to obtain a 30 or $50 \%$ drug effect with $80 \%$ power for $p<0.05$. All analyses were carried out with SigmaPlot 12.0 software (Systat, Chicago, IL). Significance level was set at $p<0.05$. Statistically significant outliers were excluded according to Grubb's test for outliers [35].

\section{RESULTS}

Social approach task

Experiment 1: Using wire cups as containments 1. 3-4 month old mice

1.1. Habituation to the chambers, no mice or object present

Mice tested at 3-4 months of age in the social approach task were not tested again at 7-8 months in this task. Mice were first habituated to the center chamber, with no access to the side chambers (phase I-see 
methods) and then the separating doors were removed to allow the mouse free exploration of all three chambers (phase II).

Two way repeated measures ANOVA revealed no main effect of genotype $(\mathrm{F}(1,44)=0.796, p=0.382)$ but a main effect of chamber $(F(1,44)=7.144$, $p=0.014)$ on the time spent in the left and right chambers in phase II (Fig. 1A), however, there was only a non-significant trend to spend more time in the left chamber in Thy1-aSyn mice (Bonferroni post hoc test : left $-243.43 \pm 22.36$ secs, right $-170.84 \pm 24.14$ secs, $p=0.067$ ). One outlier was removed from the WT group after performing Grubb's test for outliers.

\subsection{Phase III - preference for novel mouse vs. novel object}

\subsubsection{Number of entries}

Repeated measures ANOVA with chamber (i.e., chamber with empty wire cup vs. chamber with novel mouse inside a wire cup) as within subject factor and genotype as between subject factor, revealed no main effect of genotype $(\mathrm{F}(1,44)=0.0279, p=0.869)$ or of chamber $(\mathrm{F}(1,44)=4.222, p=0.053)$, and no interaction effect $(\mathrm{F}(1,44)=3.388, p=0.080)$ on the number of entries to the different chambers. WTs entered the chamber with novel object $4.60 \pm 0.94$ times on average and the chamber with novel mouse $6.0 \pm 0.94$ times on average while Thy1-aSyn mice entered the respective chambers $5.46 \pm 0.82$ times and $5.54 \pm 0.82$ times on average (data not shown). These data indicate that at this age, there are no motor deficits in Thy1-aSyn mice that could confound any behavioral deficits in the three chamber apparatus.

\subsubsection{Chamber time}

Repeated measures ANOVA with chamber (i.e., chamber with empty wire cup vs. chamber with novel mouse inside a wire cup) as within subject factor
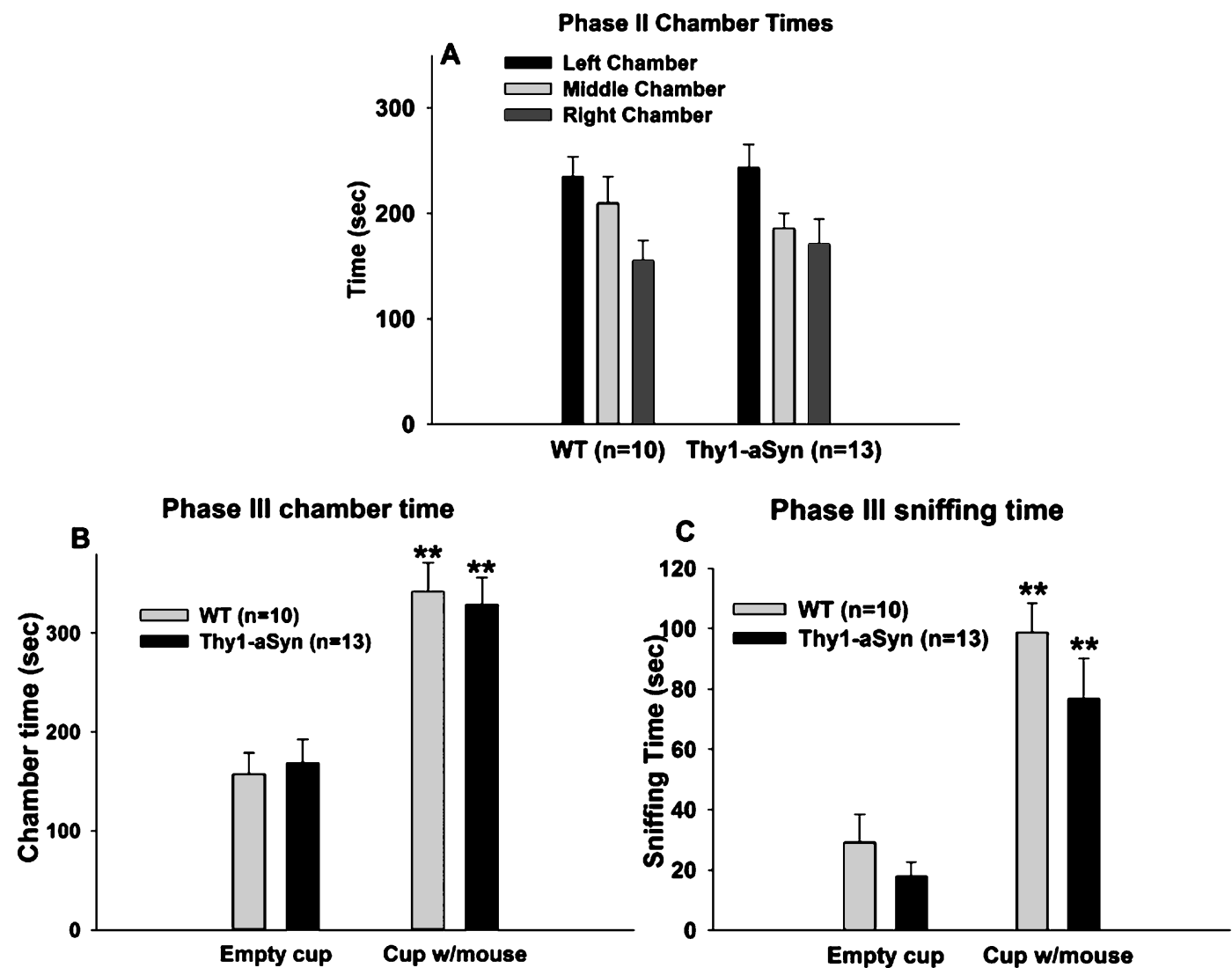

Fig. 1. Social approach task in 3-4 month old WT and Thy1-aSyn mice. (A) Times spent in the three chambers of the apparatus during the second phase of the experiment. Black bars: left chamber; light grey bars: middle chamber; dark grey bars: right chamber. (B) Times spent sniffing the empty cup (grey bars) and the cup with the novel mouse (dark bars) during the third phase of the experiment and (C) Times spent in the chamber containing the empty cup and the chamber containing the cup with the mouse (dark bars). ${ }^{* *} p<0.01$ vs. empty cup within the same genotype, repeated measure ANOVA followed by Bonferroni. WT, $n=10$; Thy 1 -aSyn, $n=13$. 
and genotype as between subject factor, revealed no main effect of genotype $(\mathrm{F}(1,44)=0.01, p=0.92)$ and did reveal an effect of chamber $(F(1,44)=22.712$, $p<0.001)$, but no interaction effect $(\mathrm{F}(1,44)=0.111$, $p=0.743)$ on the time spent in the different chambers (Fig. 1B). Comparing the time spent in the side chambers within each genotype revealed a highly significant difference for both the WT group and the Thy1-aSyn group ( $p=0.003$ ), indicating that both WT and Thy1aSyn mice at this age have a strong preference for a conspecific over an inanimate object. Indeed, Bonferroni post hoc test did not reveal any significant difference $(p=0.768)$ in the time spent in the chamber with the novel object between WT and Thy 1 -aSyn mice (WT: $n=10 ; 157.55 \pm 21.64 \mathrm{sec}$; Thy1-aSyn: $n=13$, $168.66 \pm 24.17 \mathrm{sec}$ ) or time spent in the chamber with the novel mouse (WT: $341.65 \pm 29.13 \mathrm{sec}$; Thy 1-aSyn: $328.73 \pm 27.58 \mathrm{sec} ; p=0.731$ ), indicating that there are no social deficits in Thy1-aSyn mice compared to WT mice at this age.

\subsubsection{Sniffing time}

One mouse from the WT group did not visit the side chambers in this phase. Therefore, the data for this mouse were excluded from the analysis. Repeated measures ANOVA with variable (empty wire cup vs. wire cup with a novel mouse) as within subject factor and genotype as between subject factor, revealed no main effect of genotype (Fig. $1 \mathrm{C} ; \mathrm{F}(1,44)=2.285$, $p=0.146)$ on sniffing time. There was, however, a main effect of variable $(\mathrm{F}(1,44)=51.350, p<0.001)$, suggesting a clear preference for the novel mouse regardless of genotype. There was no interaction effect between genotype and variable $(\mathrm{F}(1,44)=0.360$, $p=0.555)$ on sniffing time. Bonferroni post hoc test revealed no significant difference $(p=0.433)$ in sniffing time of the novel object between WT and Thy1-aSyn mice (WT: $n=10,29.18 \pm 9.25$ secs; Thy1-aSyn: $n=13,17.95 \pm 4.61$ secs), and also no difference in sniffing time of the novel mouse between the two genotypes (WT: $n=10,98.79 \pm 9.75$ secs; Thy1-aSyn: $n=13,76.80 \pm 13.44$ secs; $p=0.129)$. Comparing the sniffing times of novel object and novel mouse within each genotype, there was a highly significant difference within both the WT group and the Thy1-aSyn group $(p<0.001)$. Thus, testing did not reveal any social deficits in Thy1-aSyn mice at this age because they sniffed the novel object and the novel mouse the same amount of time as WTs do; furthermore, both WT and Thy1-aSyn mice have a preference for a conspecific over an inanimate object.

\section{7-8 month old mice}

Mice tested at this age in the social approach task were not tested previously at an earlier age (see methods above).

\subsection{Phase II - habituation to side chambers, no mice or objects present}

In the habituation phase, both WT and Thy1-aSyn mice spent similar times in the left and right chambers (Fig. 2A). Two way repeated measures ANOVA revealed no main effect of genotype $(\mathrm{F}(1,42)=0.118$, $p=0.731)$ or chamber $(\mathrm{F}(1,42)=0.08, p=0.78)$ on the time spent in the left and right chambers, suggesting that the mice had no preference for either one of the side chambers, similar to the younger mice.

\subsection{Phase III - preference for novel mouse vs. novel object}

\subsubsection{Number of entries}

Repeated measures ANOVA with variable (number of entries into chamber with empty wire cup vs. number of entries into chamber with novel mouse inside a wire cup) as within subject factor and genotype as between subject factor, revealed no difference between WTs and Thy1-aSyn mice (data not shown).

\subsubsection{Chamber time}

Repeated measures ANOVA with chamber (chamber with empty wire cup vs. chamber with novel mouse enclosed in a wire cup) as within subject factor and genotype as between subject factor, revealed a main effect of genotype $(\mathrm{F}(1,38)=9.768, p=0.006)$ and of chamber $(\mathrm{F}(1,38)=7.225, p=0.015)$, but no interaction effect $(\mathrm{F}(1,38)=2.038, p=0.171)$, on the time spent in the different chambers (Fig. 2B). Bonferroni post hoc test revealed a significant reduction $(p=0.027)$ of $20 \%$ in the time spent in the chamber with the novel mouse by the Thy1-aSyn mice, $(n=9 ; 254.98 \pm 12.25 \mathrm{sec})$ compared to the WT mice $(n=11 ; 314.46 \pm 17.48 \mathrm{sec})$. However, WT and Thy1aSyn did not differ in the time spent in the chamber with the novel object (WT: $216.65 \pm 19.42 \mathrm{sec}$; Thy 1aSyn: $225.02 \pm 19.24 \operatorname{secs} ; p=0.742$ ), suggesting that there is no general exploratory deficit in Thy1-aSyn mice. Note that one outlier was excluded from each group after performing Grubb's test for outliers.

Comparing the time spent in the side chambers within each genotype, there was a highly significant difference within the WT group $(p=0.007)$ but not in the Thy1aSyn group ( $p=0.407)$, indicating that the Thy1-aSyn mice do not have a preference for a conspecific over an inanimate object, as opposed to the WTs. 

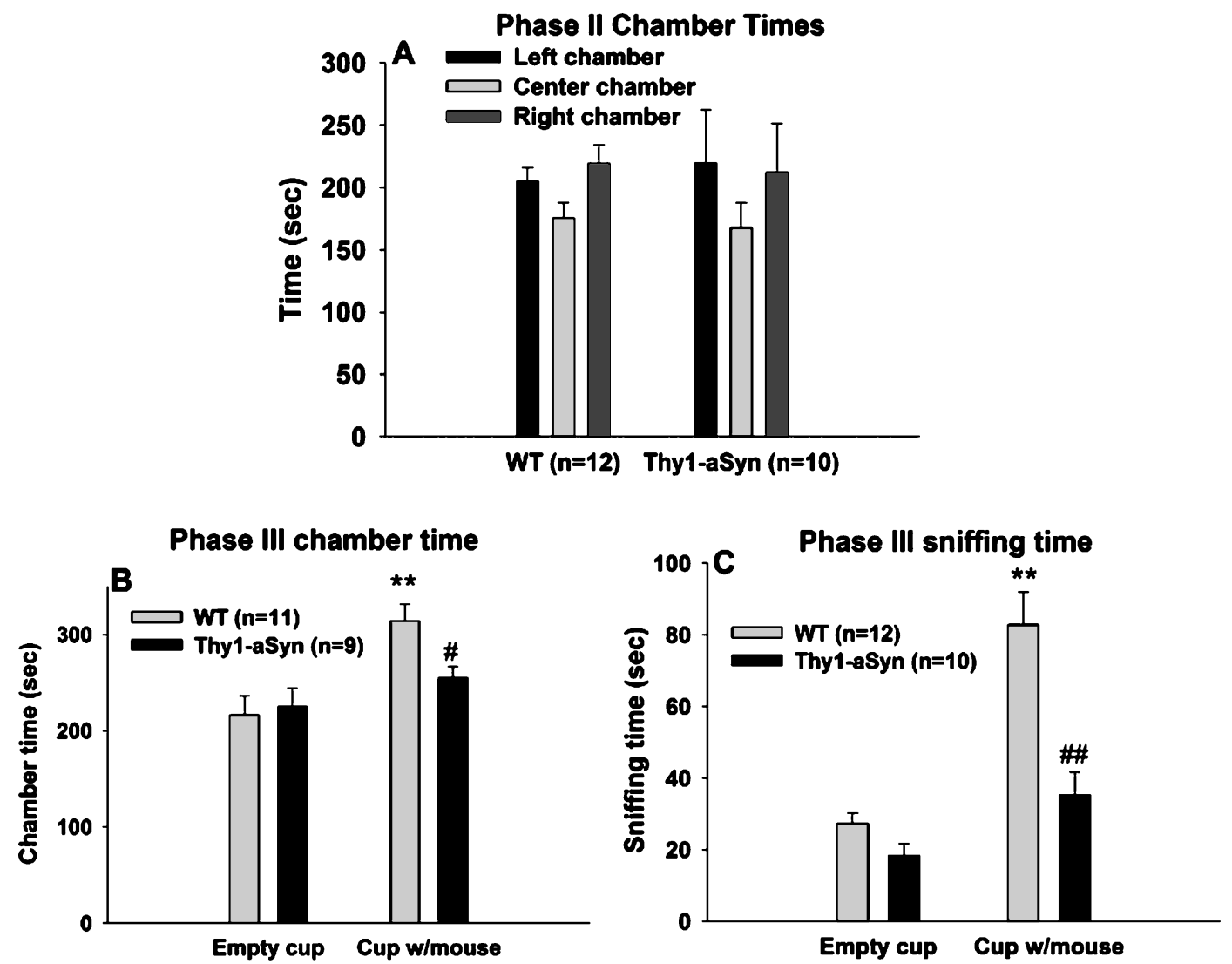

Fig. 2. Social approach task in 7-8 month old WT and Thy1-aSyn mice. (A) Times spent in the three chambers of the apparatus during the second phase of the experiment. Black bars: left chamber; light grey bars: middle chamber; dark grey bars: right chamber. WT, $n=12$; Thy $1-\mathrm{aSyn}$, $n=10$. (B) Times spent sniffing the empty cup and the cup with the novel mouse during the third phase of the experiment. WT, $n=11$; Thy 1 -aSyn, $n=9$. (C) times spent in the chamber containing the empty cup and chamber containing the cup with the mouse. WT, $n=12$; Thy 1-aSyn, $n=10$. Grey bars: WT; dark bars: Thy 1 -aSyn; ${ }^{* *} p<0.01$ vs. empty cup within the same genotype, ${ }^{\#} p<0.05,{ }^{\# \#} p<0.01$ vs. exploration time of a cup with mouse in WT, repeated measure ANOVA followed by Bonferroni.

\subsubsection{Sniffing time}

Repeated measures ANOVA with variable (empty wire cup vs. wire cup with a novel mouse) as within subject factor and genotype as between subject factor, revealed a main effect of genotype (Fig. 2C; $\mathrm{F}(1,42)=20.76, p<0.001$ ) and of variable $(\mathrm{F}(1,42)=33.14, p<0.001)$, as well as an interaction effect $(\mathrm{F}(1,42)=9.473, p=0.006)$ on sniffing time. Bonferroni post hoc test revealed a significant reduction $(p<0.001)$ of about $60 \%$ in the sniffing time of a novel mouse in the Thy1-aSyn mice $(n=10$; $35.83 \pm 5.55 \mathrm{sec})$ compared to the WT mice $(n=12$; $82.81 \pm 9.2 \mathrm{sec}$ ). However, WT and Thy1-aSyn mice did not differ in the sniffing time of a novel object, the empty wire cup (WT: $27.34 \pm 2.98$ secs; Thy1-aSyn: $18.41 \pm 3.03 \mathrm{sec} ; p=0.318$ ), suggesting that there is no general exploratory deficit in Thy1-aSyn mice, as they spent less time exploring a conspecific but not an inanimate object.
Comparing the sniffing times of novel object and novel mouse within each genotype, there was a highly significant difference within the WT group $(p<0.001)$ but not within the Thy1-aSyn group $(p=0.085)$, indicating that the WT mice have a preference for a conspecific over an inanimate object while Thy 1-aSyn do not.

These results suggest that the sociability deficits in Thy1-aSyn are progressive, and in addition, they do not result from olfactory deficits, which are already present at 3-4 months of age, when social recognition deficits were not observed [31].

\subsection{Experiment 2 - using beakers instead of wire cups as a containment tool}

As an additional control to confirm that the sociability deficits do not result exclusively from olfactory deficits, experiments were repeated with the object placed within a closed beaker to reduce a potential 

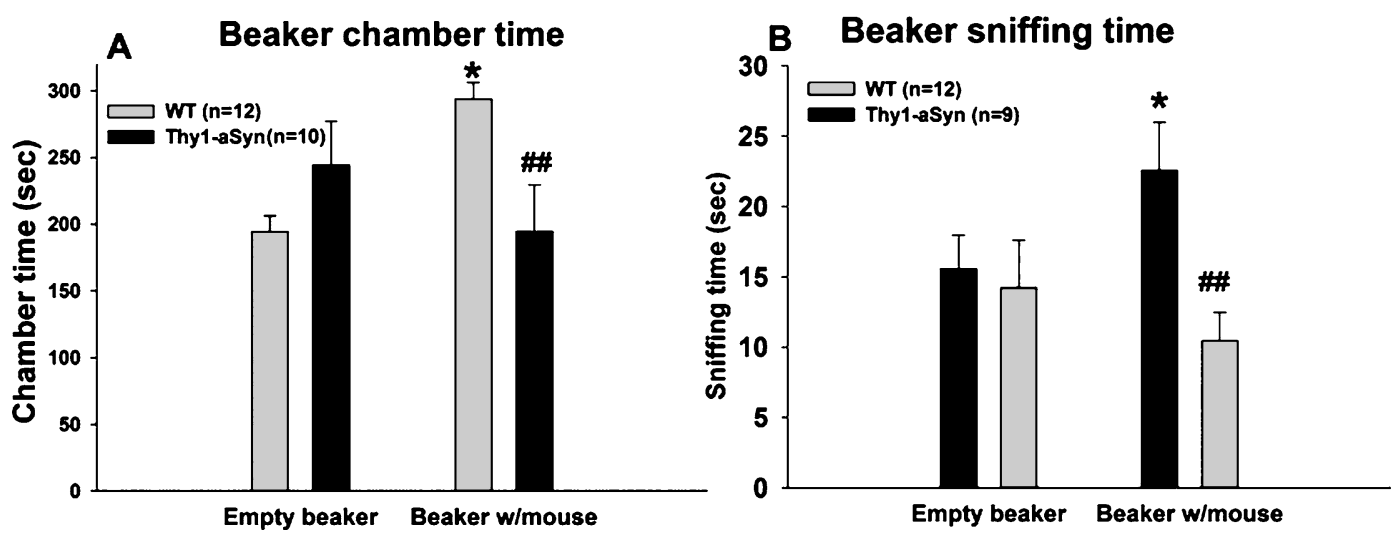

Fig. 3. Social approach task in 7-8 month old WT and Thy1-aSyn mice using a beaker as containment. (A) Times spent sniffing the empty beaker and the beaker with the novel mouse. WT, $n=12$; Thy1-aSyn, $n=10$. (B) Times spent in the chamber containing the empty beaker and the chamber containing the beaker with the mouse. WT, $n=12$; Thy1-aSyn, $n=9$. Grey bars: WT; dark bars: Thy 1 -aSyn; ${ }^{* *} p<0.01$ vs. empty cup within WT, ${ }^{\#} p<0.01$ vs. exploration time of a beaker with mouse within WT, repeated measure ANOVA followed by Bonferroni.

effect of odors. Repeated measures ANOVA with chamber (chamber with empty beaker vs. chamber with novel mouse in a beaker) as within subject factor and genotype as between subject factor, was performed on the time spent in each of the side chambers when the mouse was enclosed in the beaker and when an empty beaker was present in the other chamber (Fig. 3A). A main genotype effect was revealed $(\mathrm{F}(1,42)=7.141, p=0.015)$, with no main effect of chamber $(\mathrm{F}(1,42)=0.603, p=0.447)$. There was also an interaction effect $(\mathrm{F}(1,42)=5.436$, $p=0.030$ ). Bonferroni post hoc test revealed a significant reduction $(p=0.007)$ in the time spent in the chamber with novel mouse in the Thy1-aSyn mice $(n=10 ; 194.59 \pm 34.73 \mathrm{sec})$, compared to the WT mice $(n=12 ; 293.90 \pm 12.13 \mathrm{sec})$. In contrast, WT and Thy1-aSyn did not differ in the time spent in the chamber with the empty beaker (WT: $194.46 \pm 11.87 \mathrm{sec}$; Thy1-aSyn: $244.342 \pm 32.59 \mathrm{sec}$ ).

A comparison of the time spent in the chamber with a novel mouse vs. the chamber with an empty beaker revealed that there was a significant difference within the WT group $(p=0.032)$ but not in the Thy1aSyn group $(p=0.305)$, indicating that WT mice have a preference for a conspecific over an inanimate object, even in the absence of olfactory cues, while Thy 1-aSyn do not.

Repeated measures ANOVA with chamber as within subject factor and genotype as between subject factor revealed no main effects of genotype $(\mathrm{F}(1,40)=3.288$, $p=0.086)$ or variable $(\mathrm{F}(1,40)=0.67, p=0.423)$ on the sniffing time, but a significant interaction effect $(\mathrm{F}(1,40)=7.449, p=0.013)$. Bonferroni test revealed that WT and Thy1-aSyn mice differed from each other in sniffing the beaker containing the mouse $(22.57 \pm 3.44$ secs vs. $10.45 \pm 2.04$ secs, respectively, $p=0.007)$ but not the empty beaker $(15.56 \pm 2.41$ secs vs. $14.22 \pm 3.39$ secs, respectively, $p=0.751)$. Moreover, WT mice showed preference for sniffing the novel mouse contained in a beaker ( $p=0.014$ vs. empty beaker) whereas Thy1-aSyn did not $(p=0.221)$ (Fig. 3B). One outlier was excluded from the Thy1-aSyn group after performing Grubbs test for outliers.

These data suggest that even when olfactory cues are minimized, Thy1-aSyn mice still show sociability deficits, indicating that social cognition is defective in these animals independently from olfactory cues.

\section{Social reciprocal interactions}

Preliminary data from the social reciprocal interaction test at 7-8 month old mice indicated that total interaction time and the number of push/crawl bouts and wrestling bouts showed the greatest genotype differences at this age. Therefore, only these parameters will be described below. One cohort of mice ( $n=11$ WT and 13 Thy1-aSyn) was repeatedly tested at both 3-4 and 7-8 months, and at each age an additional cohort was used $(n=10$ per genotype at 3-4 months, $n=12 \mathrm{WT}$ and 10 Thy1-aSyn at 7-8 months - see methods). At each age, data from the two cohorts that were tested were combined and analyzed; therefore, two-way ANOVA, and not repeated measure ANOVA, was used to analyze the age differences. 


\section{Total interaction time}

Two-way ANOVA was used to compare total interaction time between ages and genotypes. A main effect of genotype was observed on total interaction time (two way ANOVA: $\mathrm{F}(1,38)=12.3, p=0.001$ ), without a main effect for age $(\mathrm{F}(1,38)=0.544, p=$ $0.465)$ or interaction $(\mathrm{F}(1,38)=2.12, p=0.154)$. At 3-4 months of age, total interaction time decreased non-significantly in pairs of Thy1-aSyn mice (Fig. 4A, $209.14 \pm 38.1 \mathrm{sec}, n=10)$ when compared with pairs of WT mice (295.52 $\pm 42.9 \mathrm{sec}, n=10 ; p=0.155)$. However, total interaction time decreased by almost three-fold in pairs of Thy1-aSyn mice $(116.9 \pm 33.24$ sec, $n=10$ ) when compared with pairs of WT mice at the age of 7-8 months $(325.7 \pm 51.75 \mathrm{sec}, n=10$; $p=0.001$ ), again indicating an age-related deficit in social behavior in Thy1-aSyn mice. We can exclude the possibility that these differences are related to a general hypoactivity, as Thy1-aSyn mice actually show hyperactivity at 7-8 months [36].

\section{Push/crawl bouts}

The number of push-crawl bouts was affected by both genotype (two way ANOVA: $\mathrm{F}(1,38)=7.7$, $p=0.009)$, and age $(\mathrm{F}(1,38)=20.8, p<0.001)$ but not by interaction $(\mathrm{F}(1,38)=2.247, p=0.143)$. Similar to total interaction time, differences between the genotypes were observed only at 7-8 months (Fig. 4B, $12.8 \pm 2.47$ bouts in WT mice, compared to $6.1 \pm 1.56$ bouts in Thy1-aSyn mice, $p=0.005)$, but not at 3-4 months of age ( $3.3 \pm 1$ bouts in WT mice, compared to $1.3 \pm 0.45$ bouts in Thy 1 -aSyn mice, $p=0.37$ ). The main effect of age resulted from an age- dependent increase in push/crawl bouts in both WT mice $(p<0.001)$ and Thy $1-a S y n$ mice $(p=0.037)$.

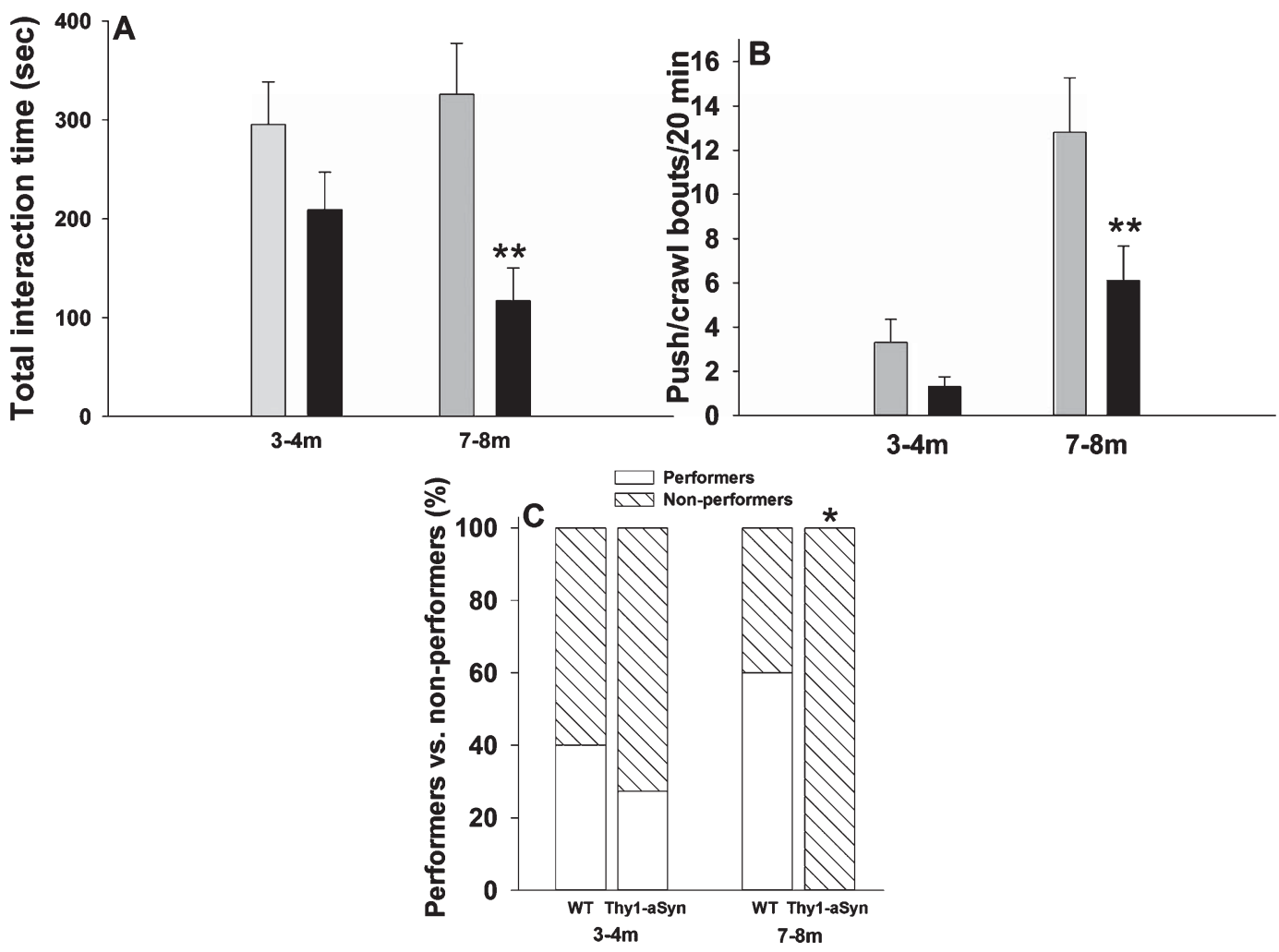

Fig. 4. Reciprocal social interactions in pairs of 3-4 and 7-8 month old WT and Thy1-aSyn mice. (A) Total interaction time (B) number of push-crawl bouts. Grey bars: WT ( $n=10$ pairs at both ages); dark bars: Thy1-aSyn ( $n=11$ pairs at $3-4$ months, 10 pairs at $7-8$ months); ** $p<0.01$ vs. age-matched WT, two-way ANOVA followed by Bonferroni. (C) Percentage of pairs displaying wrestling behavior (white portion of bar) vs. pairs not displaying this kind of behavior (hatched portion of bar). $n=10$ per group, except for Thy1-aSyn mice in wrestling bouts at $3-4$ months $(n=11){ }^{*} p<0.05$ vs. age-matched WT, Fisher's exact test. 


\section{Wrestling bouts}

Because wrestling behavior was not observed at all in Thy1-aSyn mice at 7-8 months of age, we reasoned that it would be more informative to look at this behavior qualitatively, i.e., to assess the percentage of mice displaying wrestling behavior (i.e., engaged in at least one bout of wrestling behavior) in each group rather than comparing the number of wrestling bouts. At 3-4 months of age, no differences were observed between WT and Thy1-aSyn mice: $40 \%$ of the former group (4/10) and $27.3 \%$ (3/11) of the latter group displayed wrestling behavior (Fig. 4C, Fisher's exact test: $p=0.66)$. However, by 7-8 months of age, none of the Thy1-aSyn displayed wrestling behavior in contrast to $60 \%(6 / 10)$ of the WT mice (Fig. 4C, Fisher's exact test: $p=0.01)$. As mentioned earlier, at this age mice only show deficits in motor coordination but not gross motor deficits and therefore it is unlikely that the deficit in wrestling behavior could be related to motor disability.

\section{Power analysis}

Table 2 presents the minimal number of mice needed to obtain a 30 and $50 \%$ drug effect with $80 \%$ power and a significance level of $p<0.05$. Experiments using 20-25 mice per group, which is highly practical for this kind of behavioral testing, would be adequately powered to detect a $50 \%$ improvement in social approach tasks and social reciprocal interactions, respectively, indicating that at least some of the deficits detected in this study are amenable to preclinical drug testing in this model.

\section{DISCUSSION}

To our knowledge, the present study is the first to demonstrate in a mouse model of synucleopathy [29] a range of progressive deficits in social cognition that are reminiscent of the ToM deficits reported in PD patients. These impairments were not exclusively due to olfactory deficits since they did not emerge when olfactory deficits are present [31] and persist when olfactory cues are obliterated. They were also independent from the previously described hypokinesia and striatal dopamine loss, which occur only around 14 months of age in these mice [36]. Motor deficits in coordination and fine motor skills emerge at an early age in these mice but only when mice are submitted to challenging conditions such as walking on a grid or turning on a pole $[30,37]$ and are very unlikely to play a role in the tasks used in this study.

Although the sociability deficits we observed did not primarily involve olfactory deficits, they could result from impairments in other sensory modalities such as vision. However, Thy1-aSyn mice have intact visual ability at $4-5$ months of age, as they were able to detect a light cue used in an operant reversal learning task [23]. In addition, Thy1-aSyn explored the novel object (empty cup) to the same extent as WT mice, which would be unexpected if they were visually impaired. Impaired ultrasonic vocalization, which was documented in Thy1-aSyn mice [38], is unlikely to underlie the sociability deficits, since Thy1-aSyn mice usually use chirping as a mean of communication instead of ultrasonic vocalization, but no chirping was observed during the test, and vocalization impairments are already present at 3-4 months, while sociability deficits were not.

The deficits were observed several months before the onset of striatal dopamine loss in this model [36]. This is consistent with observations in PD patients that ToM deficits are not responsive to L-dopa treatment, suggesting that they are due to non-dopaminergic mechanisms [4]. What these mechanisms are remains unknown, however, observations that ToM deficits are correlated with impairments in executive function in PD patients may provide some clues [5, 39-44]. Indeed, the frontostriatal-prefrontal circuit, which is involved in ToM in humans [45], likely mediates the mild cognitive deficits that can be observed in patients at early stages of the disease [9, 46, 47]. Alterations in this circuit, previously documented in Thy1-aSyn mice [24, 25], may cause a decreased interest in social stimuli. Alternatively, alterations in mGluR5, a receptor strongly implicated in social recognition

Table 2

Power analysis calculated for the tests described in the study: minimal number of mice needed to detect a $30 \%$ and a $50 \%$ drug effect with a power of $80 \%$ and $p<0.05$

\begin{tabular}{|c|c|c|c|c|}
\hline Behavioral test & Age & Endpoint measure & $30 \%$ effect & $50 \%$ effect \\
\hline \multirow[t]{2}{*}{ Social approach task } & $7-8$ months & Mouse sniffing time & $>30$ & 20 \\
\hline & & Chamber time & 5 & 3 \\
\hline \multirow[t]{3}{*}{ Social reciprocal interactions } & 7-8 months & Total interaction time & $>100$ & $>30$ \\
\hline & & Push/crawl bouts & $>30$ & 25 \\
\hline & & Wrestling bouts & Not applical & -aSyn was 0 \\
\hline
\end{tabular}


deficits in mouse models of ASD [28], may play a role in ToM deficits observed in patients and social recognition alterations in alpha-synuclein over-expressors. Indeed, mGluR5 receptors are elevated in the striatum of PD patients and in the cortex, hippocampus and striatum of Thy1-aSyn mice [26]. Furthermore, experiments in mouse tissue revealed a direct interaction between alpha-synuclein and mGluR5, and an increase in downstream signaling of the receptor, supporting an increased function. Finally, cholinergic pathways may contribute to ToM deficits as well. Thy1-aSyn mice have a lower level of acetylcholine in their cerebral cortex (but not hippocampus) at 6 months of age [23] and cholinergic denervation has been shown to impair social interaction [48]. Obtaining pharmacological evidence to identify which mechanism is involved by using pharmacological agents to reverse the deficits are planned for future studies but necessarily involve an extensive set of experiments that are beyond the scope of the present report.

In summary, the present study demonstrates that a genetic mouse model of pre-manifest PD reproduces social cognition deficits that have been detected in patients in early stages of the manifest phase of the disease. Together with evidence for other non-motor deficits in this model [20-22, 49], these results further support the validity of Thy 1 -aSyn mice as a platform to evaluate mechanisms and test non-dopaminergic drugs for the non-motor symptoms of PD. The three-chamber social approach task for autism research is translatable as a means of measuring social impairments in PD models. It can be instrumental in elucidating the mechanisms of ToM impairments in PD and in testing the effectiveness of developing treatment drugs.

\section{ACKNOWLEDGMENTS}

We are grateful to Dr. Constance Cummings for bringing to our attention the theory of mind deficits in PD patients. This study was funded by the Udall grant, \#PHS P50 NS38367, and by gifts to the UCLA Center for the Study of Parkinson's Disease (CSPD).

\section{CONFLICT OF INTEREST}

The authors have no conflict of interest to report.

\section{REFERENCES}

[1] Wolters E (2009) Non-motor extranigral signs and symptoms in Parkinson's disease. Parkinsonism Relat Disord, 15(Suppl 3), S6-12.
[2] Postuma RB, Aarsland D, Barone P, Burn DJ, Hawkes $\mathrm{CH}$, Oertel W, \& Ziemssen T (2012) Identifying prodromal Parkinson's disease: Pre-motor disorders in Parkinson's disease. Mov Disord, 27, 617-626.

[3] Senju A (2012) Spontaneous theory of mind and its absence in autism spectrum disorders. Neuroscientist, 18, 108-113.

[4] Roca M, Torralva T, Gleichgerrcht E, Chade A, Arevalo GG, Gershanik O, \& Manes F (2010) Impairments in social cognition in early medicated and unmedicated Parkinson disease. Cogn Behav Neurol, 23, 152-158.

[5] Yu RL, Wu RM, Chiu MJ, Tai CH, Lin CH, \& Hua MS (2012) Advanced Theory of Mind in patients at early stage of Parkinson's disease. Parkinsonism Relat Disord, 18, 21-24.

[6] Tsuruya N, Kobayakawa M, \& Kawamura M (2011) Is "reading mind in the eyes" impaired in Parkinson's disease? Parkinsonism Relat Disord, 17, 246-248.

[7] Poletti M, Enrici I, Bonuccelli U, \& Adenzato M (2011) Theory of Mind in Parkinson's disease. Behav Brain Res, 219 , 342-350.

[8] Hughes LE, Altena E, Barker RA, \& Rowe JB (2013) Perseveration and choice in Parkinson's disease: The impact of progressive frontostriatal dysfunction on action decisions. Cereb Cortex, 23, 1572-1581.

[9] Jokinen P, Karrasch M, Bruck A, Johansson J, Bergman J, \& Rinne JO (2013) Cognitive slowing in Parkinson's disease is related to frontostriatal dopaminergic dysfunction. J Neurol Sci, 329, 23-28.

[10] Schendan HE, Tinaz S, Maher SM, \& Stern CE (2013) Frontostriatal and mediotemporal lobe contributions to implicit higher-order spatial sequence learning declines in aging and Parkinson's disease. Behav Neurosci, 127, 204-221.

[11] Shine JM, Moustafa AA, Matar E, Frank MJ, \& Lewis SJ (2013) The role of frontostriatal impairment in freezing of gait in Parkinson's disease. Front Syst Neurosc, 7, 61.

[12] Ventre-Dominey J, Bourret S, Mollion H, Broussolle E, \& Dominey PF (2014) Dissociable dorsal and ventral frontostriatal working memory circuits: Evidence from subthalamic stimulation in Parkinson's disease. Hum Brain Mapp, 35, 552-566.

[13] McFarlane HG, Kusek GK, Yang M, Phoenix JL, Bolivar VJ, \& Crawley JN (2008) Autism-like behavioral phenotypes in BTBR T+tf/J mice. Genes Brain Behav, 7, 152-163.

[14] Lesage S, \& Brice A (2009) Parkinson's disease: From monogenic forms to genetic susceptibility factors. Hum Mol Genet, 18, R48-R59.

[15] Ritz B, Rhodes SL, Bordelon Y, \& Bronstein J (2012) alphaSynuclein genetic variants predict faster motor symptom progression in idiopathic Parkinson disease. PLoS One, 7, e36199.

[16] Breydo L, Wu JW, \& Uversky VN (2012) Alpha-synuclein misfolding and Parkinson's disease. Biochim Biophys Acta, 1822, 261-285.

[17] Spillantini MG, Schmidt ML, Lee VM, Trojanowski JQ, Jakes $\mathrm{R}$, \& Goedert M (1997) Alpha-synuclein in Lewy bodies. Nature, 388, 839-840.

[18] Braak H, \& Del Tredici K (2009) Neuroanatomy and pathology of sporadic Parkinson's disease. Adv Anat Embryol Cell Biol, 201, 1-119.

[19] Jellinger KA (2011) Synuclein deposition and non-motor symptoms in Parkinson disease. J Neurol Sci, 310, 107-111.

[20] Chesselet MF, Richter F, Zhu C, Magen I, Watson MB, \& Subramaniam SR (2012) A progressive mouse model of Parkinson's disease: The Thy1-aSyn ("Line 61") mice. Neurotherapeutics, 9, 297-314. 
[21] McDowell KA, Shin D, Roos KP, Chesselet MF (2014) Sleep Dysfunction and EEG Alterations in Mice Overexpressing Alpha-Synuclein. J Parkinsons Dis, 4, 531-539.

[22] Fleming SM, Jordan MC, Mulligan CK, Masliah E, Holden JG, Millard RW, Chesselet MF, \& Roos KP (2013) Impaired baroreflex function in mice overexpressing alpha-synuclein. Front Neurol, 4, 103.

[23] Magen I, Fleming SM, Zhu C, Garcia EC, Cardiff KM, Dinh D, De La Rosa K, Sanchez M, Torres ER, Masliah E, Jentsch JD, \& Chesselet MF (2012) Cognitive deficits in a mouse model of pre-manifest Parkinson's disease. Eur J Neurosci, 35, 870-882.

[24] Watson JB, Hatami A, David H, Masliah E, Roberts K, Evans CE, \& Levine MS (2009) Alterations in corticostriatal synaptic plasticity in mice overexpressing human alpha-synuclein. Neuroscience, 159, 501-513.

[25] Wu N, Joshi PR, Cepeda C, Masliah E, \& Levine MS (2010) Alpha-synuclein overexpression in mice alters synaptic communication in the corticostriatal pathway. J Neurosci Res, $\mathbf{8 8}$, 1764-1776.

[26] Price DL, Rockenstein E, Ubhi K, Phung V, MacLean-Lewis N, Askay D, Cartier A, Spencer B, Patrick C, Desplats P, Ellisman MH, \& Masliah E (2010) Alterations in mGluR5 expression and signaling in Lewy body disease and in transgenic models of alpha-synucleinopathy-implications for excitotoxicity. PLoS One, 5, e14020.

[27] Delmonte S, Gallagher L, O'Hanlon E, McGrath J, \& Balsters JH (2013) Functional and structural connectivity of frontostriatal circuitry in Autism Spectrum Disorder. Front Hum Neurosci, 7, 430.

[28] Silverman JL, Smith DG, Rizzo SJ, Karras MN, Turner SM, Tolu SS, Bryce DK, Smith DL, Fonseca K, Ring RH, \& Crawley JN (2012) Negative allosteric modulation of the mGluR5 receptor reduces repetitive behaviors and rescues social deficits in mouse models of autism. Sci Transl Med, $\mathbf{4}$, 131 ra151.

[29] Rockenstein E, Mallory M, Hashimoto M, Song D, Shults CW, Lang I, \& Masliah E (2002) Differential neuropathological alterations in transgenic mice expressing alpha-synuclein from the platelet-derived growth factor and Thy-1 promoters. J Neurosci Res, 68, 568-578.

[30] Fleming SM, Salcedo J, Fernagut PO, Rockenstein E, Masliah E, Levine MS, \& Chesselet MF (2004) Early and progressive sensorimotor anomalies in mice overexpressing wild-type human alpha-synuclein. J Neurosci, 24, 9434-9440.

[31] Fleming SM, Tetreault NA, Mulligan CK, Hutson CB, Masliah E, \& Chesselet MF (2008) Olfactory deficits in mice overexpressing human wildtype alpha-synuclein. Eur J Neurosci, 28, 247-256.

[32] Fernagut PO, Hutson CB, Fleming SM, Tetreaut NA, Salcedo J, Masliah E, \& Chesselet MF (2007) Behavioral and histopathological consequences of paraquat intoxication in mice: Effects of alpha-synuclein over-expression. Synapse, 61, 991-1001.

[33] Bolivar VJ, Walters SR, \& Phoenix JL (2007) Assessing autism-like behavior in mice: Variations in social interactions among inbred strains. Behav Brain Res, 176, 21-26.

[34] Yang M, Bozdagi O, Scattoni ML, Wohr M, Roullet FI, Katz AM, Abrams DN, Kalikhman D, Simon H, Woldeyohannes L, Zhang JY, Harris MJ, Saxena R, Silverman JL, Buxbaum JD,
\& Crawley JN (2012) Reduced excitatory neurotransmission and mild autism-relevant phenotypes in adolescent Shank3 null mutant mice. J Neurosci, 32, 6525-6541.

[35] Grubbs FE (1969) Procedures for Detecting Outlying Observations in Samples. Technometrics, 11, 1-21.

[36] Lam HA, Wu N, Cely I, Kelly RL, Hean S, Richter F, Magen I, Cepeda C, Ackerson LC, Walwyn W, Masliah E, Chesselet MF, Levine MS, \& Maidment NT (2011) Elevated tonic extracellular dopamine concentration and altered dopamine modulation of synaptic activity precede dopamine loss in the striatum of mice overexpressing human alphasynuclein. J Neurosci Res, 89, 1091-1102.

[37] Fleming SM, Salcedo J, Hutson CB, Rockenstein E, Masliah E, Levine MS, \& Chesselet MF (2006) Behavioral effects of dopaminergic agonists in transgenic mice overexpressing human wildtype alpha-synuclein. Neuroscience, 142, 12451253.

[38] Grant LM, Richter F, Miller JE, White SA, Fox CM, Zhu C, Chesselet MF, \& Ciucci MR (2014) Vocalization deficits in mice over-expressing alpha-synuclein, a model of premanifest Parkinson's disease. Behav Neurosci, 128, 110-121.

[39] Narme P, Bonnet AM, Dubois B, \& Chaby L (2011) Understanding facial emotion perception in Parkinson's disease: The role of configural processing. Neuropsychologia, 49, 3295-3302.

[40] Narme P, Bonnet AM, Dubois B, Chaby L. Understanding facial emotion perception in Parkinson's disease: The role of configural processing. Neuropsychologia 49, 3295-3302.

[41] Eddy CM, Beck SR, Mitchell IJ, Praamstra P, \& Pall HS (2013) Theory of mind deficits in Parkinson's disease: A product of executive dysfunction? Neuropsychology, 27, 37-47.

[42] Peron J, Grandjean D, Le Jeune F, Sauleau P, Haegelen C, Drapier D, Rouaud T, Drapier S, \& Verin M (2009) Recognition of emotional prosody is altered after subthalamic nucleus deep brain stimulation in Parkinson's disease. Neuropsychologia, 48, 1053-1062.

[43] Saltzman J, Strauss E, Hunter M, \& Archibald S (2000) Theory of mind and executive functions in normal human aging and Parkinson's disease. J Int Neuropsychol Soc, 6, 781-788.

[44] Mengelberg A, \& Siegert RJ (2003) Is theory-of-mind impaired in Parkinson's disease? Cogn Neuropsychiatry, 8 , 191-209.

[45] Bodden ME, Dodel R, \& Kalbe E (2010) Theory of mind in Parkinson's disease and related basal ganglia disorders: A systematic review. Mov Disord, 25, 13-27.

[46] de la Fuente-Fernandez R (2012) Frontostriatal cognitive staging in Parkinson's disease. Parkinsons Dis, 2012, 561046.

[47] Lewis SJ, Dove A, Robbins TW, Barker RA, \& Owen AM (2003) Cognitive impairments in early Parkinson's disease are accompanied by reductions in activity in frontostriatal neural circuitry. J Neurosci, 23, 6351-6356.

[48] Savage S, Kehr J, Olson L, \& Mattsson A (2011) Impaired social interaction and enhanced sensitivity to phencyclidine-induced deficits in novel object recognition in rats with cortical cholinergic denervation. Neuroscience, 195, 60-69.

[49] Hallett PJ, McLean JR, Kartunen A, Langston JW, \& Isacson O (2012) alpha-Synuclein overexpressing transgenic mice show internal organ pathology and autonomic deficits. Neurobiol Dis, 47, 258-267. 